\title{
Roll waves in floods on inclines
}

\author{
P. Bohórquez * \\ Universidad de Málaga, E.T.S. Ingenieros Industriales, Málaga, Spain
}

The unstable nature of kinematic waves, resulting from outburst floods on steep inclines, is investigated theoretically using the shallow water equations. A numerical experiment is designed in order to efficiently perform a stability analysis. By analysing the linear and non-linear numerical evolution of small perturbations, it is possible to reveal how the nature of the ensuing flow depends not only on the Froude number but also on non-parallel and time-varying characteristics of the background flow. It is also demonstrated that these effects stabilize turbulent roll-waves and raise the critical Froude number required for instability.

\section{INTRODUCTION}

Our goal in the current article is to establish the existence of physical instabilities in floods on steep inclines. To gain some inroads into this problem, we shall consider turbulent flows governed by the one-dimensional shallow-water equations. We shall limit our study to large times, in which the suddenly released, fixed mass of fluid lengthens and reaches a kinematic state.

The spreading of a suddenly released mass of fluid over an inclined plane bed has been widely studied in the above conditions [see, for instance, Weir (1983), Hunt (1984), Pritchard (2005)], and it is of hydraulic interest not only for the hydrodynamic but also for the associated transport of sediments. However, none of the previous studies have taken into account the strongly unstable character of this flow (Bohorquez 2006).

Another point of interest to be noted is that nowadays studies on the onset of instability, performed with the one-dimensional shallow-water equations, have been limited to steady flows over plane beds [e.g., Prokopiou et al. (1991)] or with spatially periodic bottom topography (Balmforth \& Mandre 2004). Many complications are introduced by non-parallel and time-dependent effects of the background state, which enrich the dynamics of real flows, when analysing its linear stability (Schmid 2007). Hence, the author considers that the study about this time-varying space-dependent system is an outstanding issue.

The reader can find in this volume the definition of the dam-break problem for a constant but arbitrary slope of the bottom as well as the asymptotic solution under the kinematic wave approximation (Bohorquez 2007). However, when friction is taken into account in such problem, it is well known that the flow loses memory of its initial distribution. Hence, considering a point source of mass, located at $X=0$ with initial conditions

*This research has been supported by a fellowship from the Ministerio de Educación y Ciencia of Spain (AP-2004-3719) 
$U(X, 0)=0$ and $\eta(X, 0)=A \delta(X)$, being $A$ the released volume, allows to obtain a more simple solution. In fact, defining a scaling factor $\epsilon$, and rescaling non-dimensional variables in Bohorquez (2007), $\S 2$, by the following relations,

$$
x \equiv X \epsilon \tan \theta, \quad \tau \equiv t \epsilon \tan \theta \sqrt{(8 / f) \sin \theta}, \quad u \equiv U \sqrt{f /(8 \sin \theta)},
$$

conservation of mass and momentum read

$$
\partial_{\tau} \eta+\partial_{x}(\eta u)=0, \quad \epsilon\left[F r_{e q}^{2}\left(\partial_{\tau} u+u \partial_{x} u\right)+\partial_{x} \eta\right]=1-u^{2} / \eta,
$$

respectively, where $F r_{e q}$ is defined as $F r_{e q}^{2} \equiv(8 / f) \tan \theta$. The Froude number, which states the local ratio of the flow velocity $U$ to the wave celerity $c \equiv(\eta \cos \theta)^{1 / 2}$, is given in the new variables by $F r \equiv U / c=F r_{e q} u / \sqrt{\eta}$, and reduces to the constant parameter $F r_{e q}$ when a balance is struck between the friction of the bottom and the streamwise component of gravity, in other words, when $\epsilon$ is neglected in the momentum balance.

The set of non-dimensional equations (2) is exactly the same as those used by Hunt, but they come from different dimensional equations - since Hunt applied the St. Venant equations valid just for small slopes of the bottom. In view of this, we find that dimensionless Weir's (1983) and Hunt's (1984) solutions for floods on inclines are also valid for steep slopes when non-dimensional variables are defined correctly. This result will be used for the formulation of the stability problem (see bellow).

We start defining the linear stability equations and the base state in $\S 2$, as well as the numerical schemes used in following sections. Next, in $\S 3$, we study numerically the linear and non-linear stability of our background flow. We devote $\S 4$ to summarize our results. Overall, the study is focused on non-uniform and unsteady base flows, but some of the results carry over parallel (uniform and steady) flows, in order to check with previous analytical results the ability of the numerical schemes to reproduce the behaviour of the perturbations.

\section{FORMULATION OF THE PROBLEM AND NUMERICAL SCHEMES}

Here, we are interested in kinematic waves as base state, obtained when the parameter $\epsilon$ is small in the momentum balance (2), provided $u$ and $\eta$ as well as their gradients are order unity. In this case, the velocity and height can be expanded in powers of $\epsilon$ to find an analytical solution:

$$
u_{b}(x, \tau)=V(x, \tau)+\epsilon V_{1}(x, \tau)+O\left(\epsilon^{2}\right), \quad \eta_{b}(x, \tau)=H(x, \tau)+\epsilon H_{1}(x, \tau)+O\left(\epsilon^{2}\right) .
$$

The first term in the expansion, called first-order outer solution, was found by Hunt (1984), and is given by

$$
V(x, \tau)=2 x /(3 \tau), \quad H(x, \tau)=V^{2} .
$$

We shall carry the stability analysis on this basic flow.

To analyse the stability of such time-dependent system, we shall superimpose an infinitesimal disturbance, $v(x, \tau)$ and $h(x, \tau)$, on the background flow,

$$
u=u_{b}(x, \tau)+v(x, \tau), \quad \eta=\eta_{b}(x, \tau)+h(x, \tau),
$$

where $|h| \ll \eta_{b}$ and $|v| \ll\left|u_{b}\right|$. Substituting (5) in (2), and neglecting second order terms in the small perturbations, one is left with the following set of linear equations for the perturbations:

$$
\frac{\partial h}{\partial \tau}+\frac{\partial \eta_{b} v}{\partial x}+\frac{\partial h u_{b}}{\partial x}=0, \quad F r_{e q}^{2}\left[\frac{\partial v}{\partial \tau}+\frac{\partial v u_{b}}{\partial x}\right]+\frac{\partial h}{\partial x}=\frac{u_{b}^{2}}{\epsilon \eta_{b}}\left[\frac{h}{\eta_{b}}-\frac{2 v}{u_{b}}\right],
$$


The above partial differential equations (PDEs) require one initial condition for both the velocity $v$ and height $h$ fields. We shall consider the initial configuration (at $\tau=\tau_{0}$ ) of the perturbation $v$ on the basic flow $u_{b} \approx V$ (henceforth, we will use just the first term in the expansion (3) to compute the base state) given by

$$
v\left(x, \tau_{0}\right)= \begin{cases}B\left(l_{1}\right) \sin \left(2 \pi n \frac{x-l_{1}}{l_{2}-l_{1}}\right) & \text { if } l_{1} \leq x \leq l_{2} \\ 0 & \text { otherwise }\end{cases}
$$

where $B$ denotes the initial amplitude of the perturbation $\left(B \equiv \delta V\left(l_{1}, \tau_{0}\right)\right.$, being $\delta$ a very small parameter), $l_{1}$ and $l_{2}$ establish the boundary enclosing the initial waves, and $n$ denotes the number of waves. For the height, the relation that follows is used: $h\left(x, \tau_{0}\right)=$ $(V+v)^{2}-H \approx 2 V v$, which is $O(\delta H)$.

The convective nature of the perturbations is easily established in terms of the Froude number. This is understandable in view of the fact that both the linear (6) and nonlinear (2) system of PDEs have two real and distinct characteristic curves, and these are the same for small waves. Hence, perturbations travel along the characteristics $C_{ \pm}: d x / d \tau=u_{b} \pm$ $\eta_{b} / F r_{e q}$, and the convective nature comes for supercritical kinematic waves: $d x / d \tau \approx$ $V\left(1 \pm 1 / F r_{e q}\right)>0$ with $F r_{e q}>1$. From now on, we are interested just in supercritical flows.

To compute the linear evolution of the perturbations, we use a finite-difference scheme on an uniform grid with mesh size $\Delta x$. The time integration is performed with a CrankNicolson method, and a fourth-order central-differences scheme is applied in space - the truncation error is $O\left(\Delta \tau^{2}, \Delta x^{4}\right)$, where $\Delta \tau$ is the time step. In order to use the minimal number of nodes, we initially solve the resulting linear system of equations just in a small region $I_{1}$ (which bounds the waves) with homogeneous boundary conditions. Before the wave train travelling downwards reaches the end of $I_{1}$, a new domain $I_{2}$ is defined containing the subregion in which waves live, and it is enlarged downwards adding new nodes. This process is repeated several times during the numerical simulation. Thus, we have to solve in each time step a linear system of equations with just eleven diagonals. Band storage mode is used to minimize memory requirements. To that end, subroutines DGBMV and DLSLRB into the IMSL Numerical Libraries are used.

On the other hand, the full non-linear set of equations (2) is solved with the same numerical technique as in Bohorquez (2007). In this case, taking into account that the flow is supercritical at both inlet and outlet, two boundaries conditions are imposed upstream (given by Eq. 4), and the characteristic variable extrapolation (CVE) method is used downstream. Next, we present some results.

\section{RESULTS}

First, we will show results corresponding with the parallel case. To that end, we have considered an uniform and steady basic flow with $U=1$ and $\theta=1^{\circ}$. This flow has been initially disturbed with $n$ small waves ( $n=10$ ), and its nonlinear evolution has been computed following the approach introduced in $\S 2$. Figure 1a depicts the maximum amplitude of the wave train as a function of time for several Froude numbers. Initially, the nonlinear growth rate is in agreement with theoretical results obtained using the linear theory, but as time goes on, and the wave amplitude is not so small, the wave changes its shape towards hydraulic jumps (as shown by the inset in Fig. 1b) and saturation appears due to nonlinear effects.

One should note that the main difference of the numerical technique we have used, with respect that applied by Brook et al. (1999), are the boundary conditions. Traditionally, roll waves are numerically studied by using their periodicity properties, and periodic boundary conditions are usually stated. However, in order to analyse the stability of more 

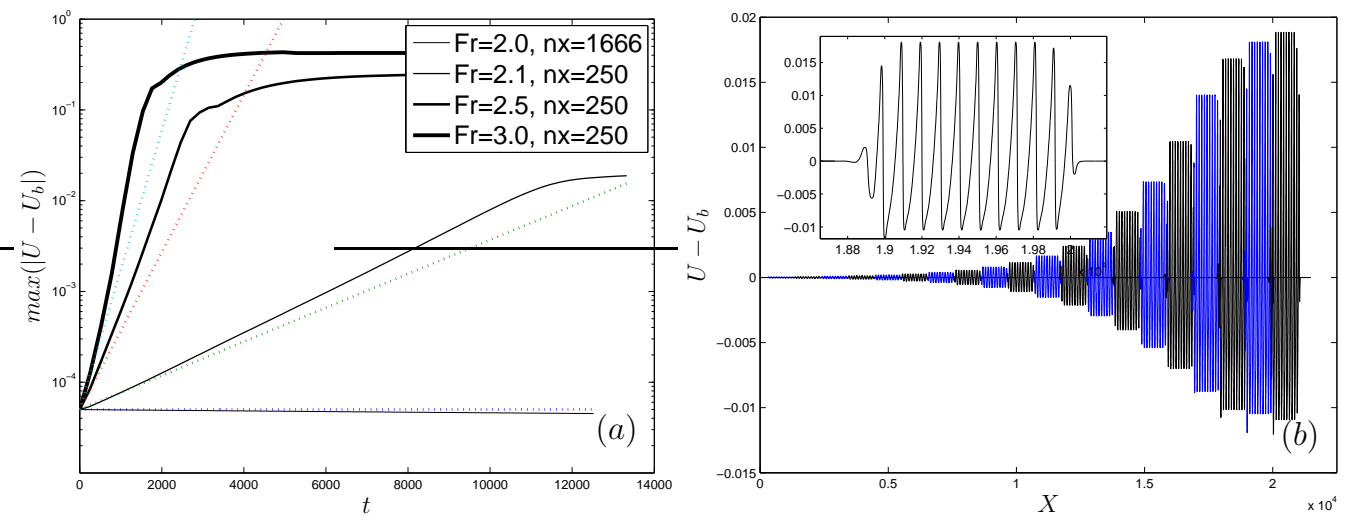

Figure 1: (a) Amplitude of the water wave velocity, $U-U_{b}$, against non-dimensional time, $t$, for different steady flow Froude numbers $F r_{e q} \geq 2.0$ and an initial amplitude of the velocity perturbation $\delta=5 \times 10^{-5}$. The solid curves represents the solution obtained from the non-linear numerical code, and the dashed lines indicate the corresponding growth rates obtained from the linear stability analysis (see, e.g., Brook et al. 1999). $n x$ represents the number of nodes used by wave. (b) Plots of perturbation velocity, $U-U_{b}$, against distance along the channel, $X$, at several instants of time $\left(t<1.4 \times 10^{4}\right)$ corresponding with the case $F r_{e q}=2.1$

general basic flows (see bellow), which are not periodic, we have followed a different approach (see $\S 2$ for details).

Next, we analyse the stability of the unsteady and non-uniform base state given by (4). One should focus the study on the question that follows: how temporal and spatial gradients of the basic flow affect the stability criteria. For simplicity, we study only the linear evolution of the perturbation, and due to the fact that the linear case conclusions do not depend on the wave amplitude $B$, we have normalized the perturbation velocity $v$ $(B=1)$. In order to present the results, we have selected a wave length equal to unity, $\lambda \equiv$ $\left(l_{2}-l_{1}\right) / n=1$, a wave train generated by three waves, $n=3$, and the initial conditions of the base state will change from one simulation to another.

The first novel result we have found, when analysing the particular case $F r_{e q}=2$, is that non-parallel and unsteady effects stabilize the flow. Inspection of Figures $2 a, b$ corroborates this statement. Furthermore, while the wave length remains constant in the parallel case as time goes on (Prokopiou et al. 1991; Brook 1999), disturbances lengthen in the present case, as it is shown in Figure $2 b$.

Second, when increasing the Froude number, the flow becomes unstable - as it is shown in Figure 2c, which corresponds with the numerical simulation of the same perturbation and base flow as in Figure 2a but rising the Froude number up to $F r_{e q}=2.2$. However, even background flows with Froude numbers larger than two are stable for some wave numbers. In fact, the numerical simulation of the case plotted in Figure $2 b$ with Froude number $F r_{e q}=2.2$, instead of $F r_{e q}=2$, reports similar effects, i.e., the amplitude decreases while the wave lengthens (we do not show the graph due to space requirements).

\section{CONCLUSIONS}

In this study we have considered the stability analysis of a suddenly released, fixed mass of fluid that reaches a kinematic regime. In particular, we have designed a numerical experiment, and a clever numerical method, that allows to compute the linear and nonlinear evolution of small perturbations.

We have found that non-uniform and unsteady effects of the background flow stabilize turbulent roll-waves and raise the critical Froude number required for instability. The 

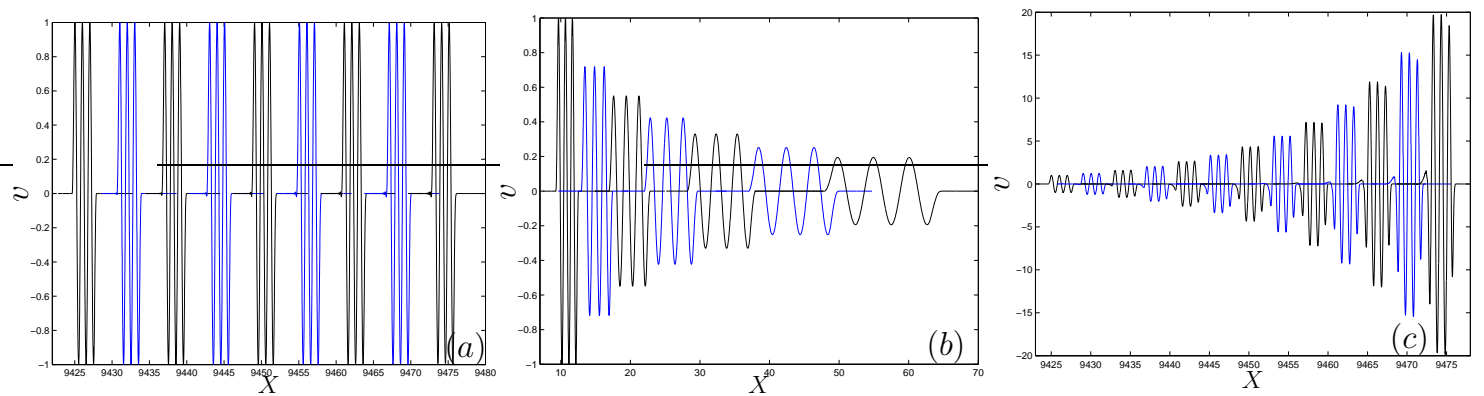

Figure 2: (a) Plots of perturbation velocity, $v$, against distance along the channel, $X$, at several instants of time with $F r_{e q}=2, \lambda=1, U_{b}\left(l_{1}, \tau_{0}\right)=.1282$, and $\partial_{X} U_{b}\left(l_{1}, \tau_{0}\right)=$ $1.36 \times 10^{-5}$. The case (b) corresponds with the case (a) but with $U_{b}\left(l_{1}, \tau_{0}\right)=3.9$ and $\partial_{X} U_{b}\left(l_{1}, \tau_{0}\right)=4.09 \times 10^{-4}$. (c) As in (a) but with $F r_{e q}=2.2$. The solution has been obtained with the linear numerical code described in $\S 2$.

well known stability criteria for parallel flows at high Reynolds number (the basic flow is unstable for any wave length and Froude numbers larger than 2) differs abruptly of that resulting from kinematic waves. One of the most noticeable effects is stabilization of disturbances on basic flows with $F r_{e q}=2$. In addition to that, for larger Froude numbers, $F r_{e q}>2$, the wave amplitude decreases or increases depending on the velocity, and both spatial and temporal gradients of the base flow. Furthermore, waves lengthen as time goes on - this behaviour is also different with respect to the parallel one, in which waves remain with constant wave length even when hydraulic jumps are developed.

P. B. gratefully acknowledges many interesting and fruitful discussions on this work with Prof. Ramón Fernández-Feria.

\section{REFERENCES}

Balmforth, N.J. \& Mandre, S. (2004). Dynamics of roll waves. J. Fluid Mech. 514, $1-33$.

Bohorquez, P. (2006). Nonparallel spatial stability of shallow water flow down an inclined plane of arbitrary slope. Series Monográficas: Trabajos de Investigación Tutelada, UMA, Málaga, Spain. (ISBN 84-690-3171-6)

Bohorquez, P. (2007). On the wave-front shape and the advancing of the wetting front of a dam-break flood over an inclined plane of arbitrary bottom slope. Numerical Modelling of Hydrodynamics for Water Resources. Taylor and Francis, /Balkema, Leiden, The Netherlands. (in press)

Brook B.S., Falle, S. \& Pedley, T.J.(1999). Numerical solution for unsteady gravitydriven flows in collapsible tubes: evolution and roll-wave instability of a steady state. J. Fluid Mech. 396, 223-256.

Hunt, B. (1984). Perturbation solution for dam-break floods. J. Hydraul. Eng. 110(8), $1058-1071$.

Pritchard, D. (2005). On fine sediment transport by a flood surge. J. Fluid Mech. 543, 239-248.

Prokopiou, Th., Cheng, M. \& Chang, H.C. (1991). Long waves on inclined films at high Reynolds number. J. Fluid Mech. 222, 665-691.

Schmid, P.J. (2007). Nonmodal stability theory. Annu. Rev. Fluid Mech. 39, 129-162.

Weir, G. J. (1983). The asymptotic behaviour of simple kinematic waves of finite volume. Proc. Roy. Soc. of London 387, 459-467. 